\title{
Localization of the HPV-16 Minor Capsid Protein L2 by Difference Imaging.
}

\author{
B.L.Trus *, C.B.Buck**, N.Cheng***, D. R. Lowy**, A.C.Steven***, J.T.Schiller** \\ * CIT, ** NCI, *** NIAMS, NIH, DHHS, Bethesda, MD 20892-5624 USA
}

Papillomaviruses are a diverse group of DNA tumor viruses that infect the skin and mucous membranes of many vertebrate species, including humans. While many papillomavirus types are associated with benign skin warts, certain mucosal human papillomavirus (HPV) types have been shown to be the necessary cause of virtually all cases of cervical cancer. Papillomavirus virions have a major capsid protein, L1, and a minor capsid protein, L2. L1 can spontaneously assemble into an isometric, icosahedral capsid, composed of 360 L1 molecules arranged in 72 pentameric capsomers. L2, which plays a key role in bringing the viral genome to the nucleus during the establishment of infection and in encapsidating the viral genome during the late stages of viral replication, is dispensible for capsid formation. L1-based virus-like particles (VLPs) are the basis of a prophylactic vaccine against cervical cancer that is currently in phase 3 clinical trials. While L1based vaccines are highly HPV type-specific, recent evidence suggests that antibody responses to L2 may confer protection against the gamut of HPV types. The arrangement and relative abundance of L2 has been unclear. Early studies of wart-derived papillomavirus virions had suggested an L1:L2 ratio of about 12 (i.e., 30 copies of L2 per virion), while later studies of recombinant VLPs suggested L1:L2 ratios closer to 30 (i.e., 12 copies of L2 per virion) [1, 2]. It has been widely assumed that monomers of L2 contribute a small ordered density to the apex of each of the 12 capsid vertices. Recent advances in methods for intracellular production of infectious VLPs composed of L1 + L2 have made it possible to revisit the issue in a more controlled fashion [3]. Detailed quantitation of HPV type 16 L1 and L2 in VLPs produced in mammalian cells show that highly purified capsids contain an average of 30 to 36 copies of L2, consistent with early analysis in vivo-derived virions. Using reconstituted GFP technology [4], we further demonstrate that the amino and carboxyl termini of adjacent L2 molecules can be apposed to within $20 \AA$ of one another.

We report the localization of L2 by difference imaging between (L1+L2) (Fig 1a) and L1-only (Fig 1b) VLPs of HPV16. A 3D reconstruction (3DR) of 1918 mature particles (Fig 2a) exhibits a typical papillomavirus structure [2], as did a 3DR of HPV16 L1-only capsids. While the surface-rendered views show little difference between the two types of particle, the central sections (Fig $2 \mathrm{~b}$ and $\mathrm{c}$ ) show additional mass underlying the capsomers in L1+L2 particles (Fig 2b). A difference section is shown in Fig 2d. After proper scaling, the L1 3DR can be subtracted from the L1+L2 3DR, showing an L2-only image (Fig 3). It is likely that the positive density seen in the difference map represents only the ordered portion of L2. The simplest interpretation of the data is that a monomer of L2 is randomly associated with half of the capsomers in the virion. This arrangement would be reminiscent of polyomaviruses, in which each capsomer lumen is occupied by a monomer of one of the minor capsid proteins, VP2 or VP3. However, alternative scenarios, such as an L2 oligomer, e.g. a trimer, associated with a randomly selected subset of capsomers, or arrangements involving simultaneous binding of L2 monomers to two or more capsomers, cannot be ruled out. An additional intriguing feature of the HPV16 VLP preparations was the presence of a sub-population of larger capsids with pronounced openings in the capsid floor (right side of Figure 4). It is likely that these larger capsids represent intermediates in the recently described process of papillomavirus capsid maturation [5]. A similar capsid closure process has been observed to occur during the maturation of HSV-1 [6]. Elucidation of the abundance and arrangement of L2, as well as further investigation of its possible exposure during maturation and cellular entry, should help inform efforts to improve L2-based vaccines against HPV and provide insight into the mechanisms underlying the biological activities of L2. 
1. Rippe et al, (1989) Virol 171:298-301

2. Trus et al. Nat. Struct. Biol. (1997) 4:413-420.

3. Buck et al. (2004) J. Virol. 78:751-7.

4. Tromans (2004) Nat. Rev. Mol. Cell Biol. 5:865

5. Buck et al. (2005) J. Virol. 79:2839-2846

6. Heymann et al., Nat. Struct. Biol. (2003) 10:334-341.

Figure 1 (a) HPV-16 VLPs containing L1+L2 from cryo-electron microscopy (b) HSV-1 VLPs containing L1 only. Bar $=100 \AA$

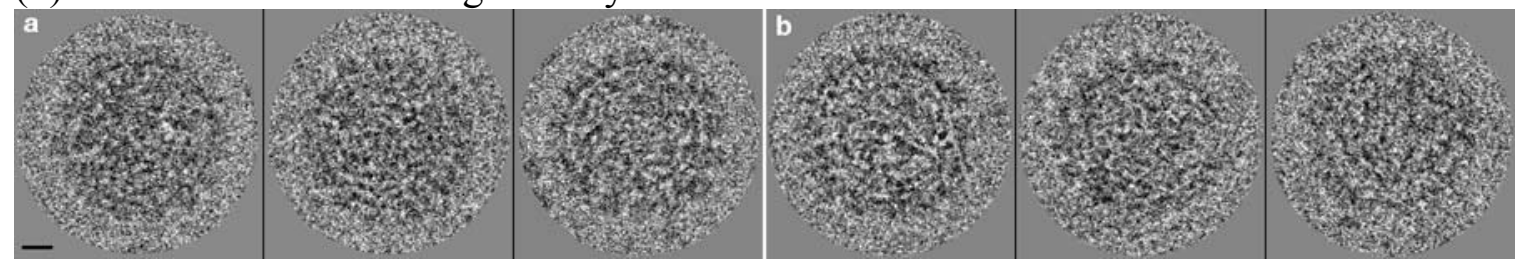

Figure 2 (a) 3D reconstruction of HSV-1 HPV-16 VPs containing L1+L2 - 2-fold view (b) central section (c) central section from L1 only 3DR. Note the additional density under the 5-fold penton in c indicated by the *. (d) Difference image of L1+L2 section minus L1 section. Bar $=100 \AA$

Figure 3 Difference imaging is used to locate the $\mathrm{L} 2$ protein. The $\mathrm{L} 1$ only $3 \mathrm{D}$ reconstruction is subtracted from the $\mathrm{L} 1+\mathrm{L} 2$ reconstruction yielding the location of $\mathrm{L} 2$ under each penton.

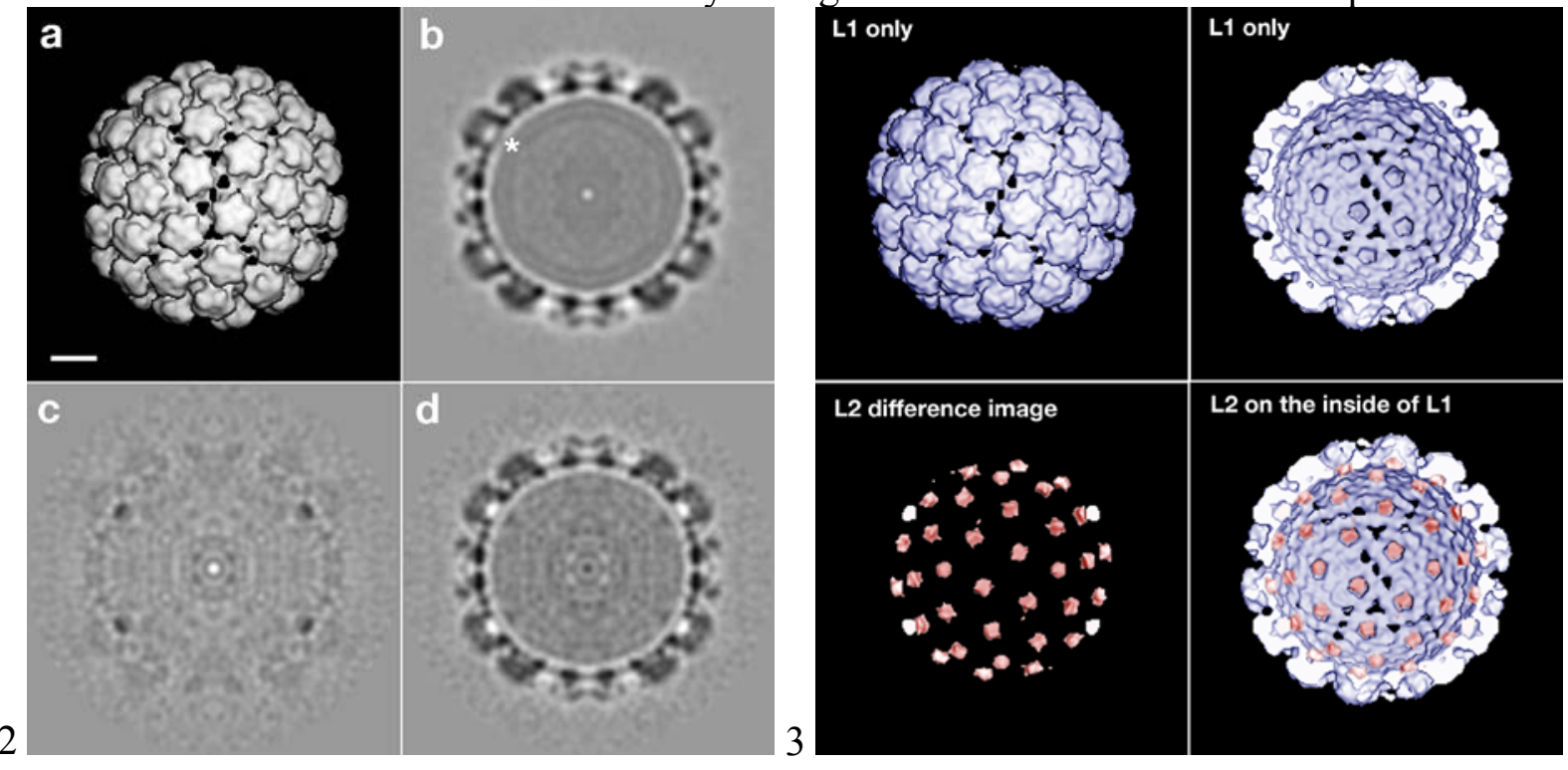

Figure 4 Size intermediates were observed in the 3D reconstruction of HSV-1 containing $\mathrm{L} 1+\mathrm{L} 2$. Left $3 \mathrm{DR}$ is a mature capsid, right most image is $8 \%$ larger.

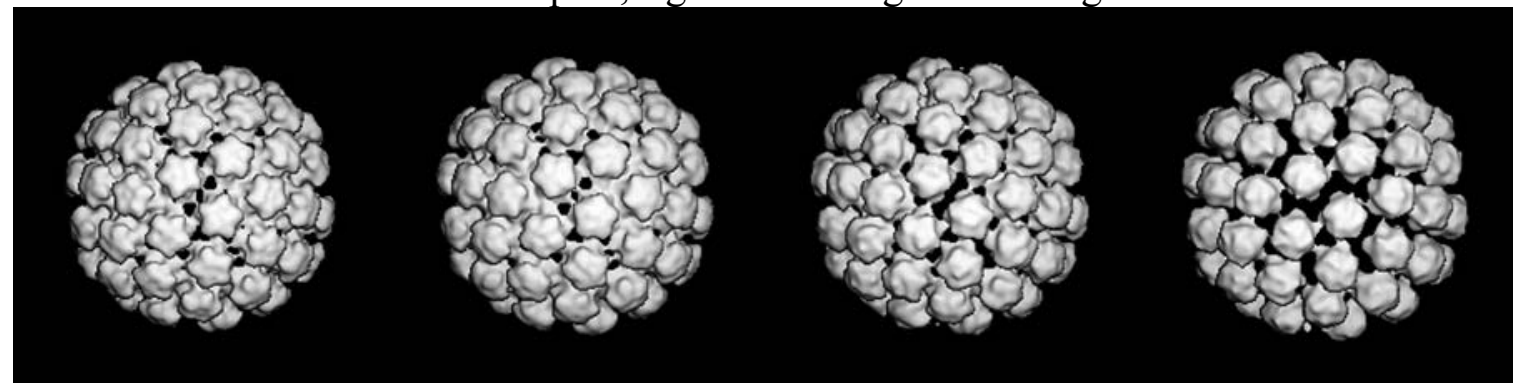

